\title{
Melanoma skin cancer detection using deep learning and classical machine learning techniques: A hybrid approach
}

\author{
Jinen Daghrir ${ }^{1,2}$, Lotfi Tlig ${ }^{2}$, Moez Bouchouicha ${ }^{3}$, Mounir Sayadi ${ }^{2}$ \\ ${ }^{1}$ Université de Sousse, ISITCom, 4011, Hammam Sousse, Tunisia \\ ${ }^{2}$ Université de Tunis, ENSIT, Laboratory SIME, Tunisia \\ ${ }^{3}$ Aix Marseille Univ, Université de Toulon, CNRS, LIS, Toulon, France
}

\begin{abstract}
Melanoma is considered as one of the fatal cancer in the world, this form of skin cancer may spread to other parts of the body in case that it has not been diagnosed in an early stage. Thus, the medical field has known a great evolution with the use of automated diagnosis systems that can help doctors and even normal people to determine a certain kind of disease. In this matter, we introduce a hybrid method for melanoma skin cancer detection that can be used to examine any suspicious lesion. Our proposed system rely on the prediction of three different methods: A convolutional neural network and two classical machine learning classifiers trained with a set of features describing the borders, texture and the color of a skin lesion. These methods are then combined to improve their performances using majority voting. The experiments have shown that using the three methods together, gives the highest accuracy level.
\end{abstract}

Index Terms-Melanoma detection, deep learning, classical machine learning, data fusion, majority voting

\section{INTRODUCTION}

Melanoma the deadliest form of skin cancer, is considered as the less common form of skin cancers but it is the most fatal. As mentioned above, it can quickly spread to other parts of the body. Melanoma arises through malignant transformation of melanocytes which are derived from the neural crest neoplasia [7]. Melanoma causes 55500 cancer deaths annually which is $0.7 \%$ of all cancer deaths. The incidence and mortality rates of melanoma differ from one country to another due to the variation of ethnic and racial groups [15]. Established risk factors for melanoma include ultraviolet radiation, life in low geographic latitudes, high alcohol consumption, consuming fatty foods, the presence of melanocytic or dysplastic naevi, a family or personal history of melanoma, phenotypic characteristics including fair hair, eye, and skin colors [13].

The incidence and mortality of melanoma are related to the development index (HDI), The increase in the HDI index increases access to health services and early detection of disease and treatment of the disease at an early stage, thereby reducing mortality [13]. Yet, statistics shows that the 5-year relative survival rate for people who has been diagnosed with melanoma in an early stage is about $98 \%$. However, about $20 \%$ to $50 \%$ of people having melanoma in advanced stage will be alive 5 years after diagnosis [15].

Since, it is important that the melanoma is caught at an early stage. Self examination is so vital even if you have carefully protected your skin from ultraviolet radiations. Thus, people should examine their skins head to toe regularly, looking for any lesions that might be turned into melanoma. Self-exams can help you identify skin cancers in an early stage, when the odds of curing them are completely high. Yet, physicians encourage people to routinely do self-examination if they notice any suspicious-looking lesions. "when in doubt, cut it out", says Darrick Antell, MD, a board-certified plastic and reconstructive surgeon practicing in New York.

In this matter, it becomes vital to use supportive imaging techniques that have been shown to improve and facilitate the diagnosis process. These techniques are build based on strategies invented by physicians to capture the melanoma at an early stage. Automatic detection of melanoma is comprised of a variety of steps including preprocessing, extraction of interested area, post-processing, and lesion inspection. These steps look like the steps of the classical pattern recognition system, where the main design steps of such system are: image acquisition, image processing and lesion segmentation, and classification methodology of the lesion in question [14]. In this paper, we present a new method for melanoma detection for a better self-examination.

In this paper, the first section gives a brief description of some existing work and the visual cues that are used by physicians for melanoma detection. The second section is reserved to introduce the proposed method. All the conducted experiments and the results evaluating the performance of the proposed method are given after that. In the last section, a discussion and conclusion are given.

\section{RELATED WORK}

One typical and commonly used clue for melanoma diagnosis is the ABCDE signs which is a useful indicator for melanoma. This feature can differentiate a malignant melanoma from a benign skin lesion based on five characteristics, namely asymmetry (A), border irregularity (B), color variability (C), diameter greater than $6 \mathrm{~mm}(\mathrm{D})$, and evolution (E) or any kind of change. Melanoma detection typically involves several processes: image acquisition and preprocessing, lesion segmentation, lesion characterisation and 
finally lesion classification. The exact order in which these processes are applied varies from one method to another, however, some of them can skip or add other processes, or use some of them in a hybrid way.

The first step in expert systems used for melanoma detection involves the acquisition of the tissue digital image. The most common techniques used for this purpose are the epiluminence microscopy(ELM), it is also known as dermoscopy [9]. Yet, smartphone cameras are becoming more and more capable and effective to capture skin lesions, the resolution of the image has improved over years which can encourage experts to use smartphone cameras to clinically imaged lesions rather than using traditional acquisition methods [11]. In addition this help people to easily do self-examination in order to inspect any suspicious lesion.

Most of the existing systems use the dermoscopic images which are proved to be very efficient in melonoma inspection. These systems can use image processing and classical machine learning techniques [11], [3], [6] or more sophisticated and powerful technique which is the deep learning [10], [18].

After the image is taken, a lesion segmentation is conducted in order to isolate the pathological skin lesions from the surrounding healthy skin. This step is considered to be critical step in the whole process of melanoma detection. One interesting and recent method have been proposed by Uzma Jamil et al. [11], which consists on separating the foreground (the lesion) from the background by filtering the image with the gradient magnitude.

Then, a set of characteristics are extracted. Generally, these primitives will represent only the ABCD rule which describes the color, the border and the texture looking for some differential structures [14]. Studying the texture of a skin lesion can bring out many characteristics which can be extracted using different kind of texture descriptors, such as SIFT feature, SURF and HOG [12]. However in [17], the authors have used color in addition to the textural features. The $\mathrm{CN}$ (Color Name) features which are linguistic color labels representing 11 different colors, are used to detect the presence of the black and blue colors in a lesion. The blue-black color is another typical feature that is used by physicians to recognize melanoma. It is defined as the presence of a combination of blue and black pigmented areas involving at least $10 \%$ of the lesion surface [2].

Once the features are extracted, a training phase is needed to create a model that differentiate between malignant and benign lesions. Researchers have widely used the support vector machines (SVMs), due to their effectiveness and low complexity in generating an accurate model [3], [8]. However, in [6] authors proposed a comparison between a K-Nearest Neighbor (KNN) classifier and Artificial Neural Network (ANN) which prove to be more accurate than the KNN, since they iteratively adjust their connection weights by minimizing the error between the ground truth and the predicted outputs. Yet, involving the concept of deep learning has become a trend in melanoma detection systems. Different from the traditional pattern recognition systems, which heavily rely on the result

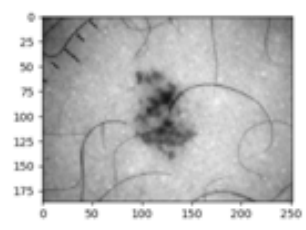

RED

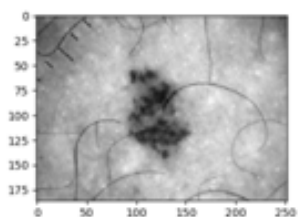

GREEN

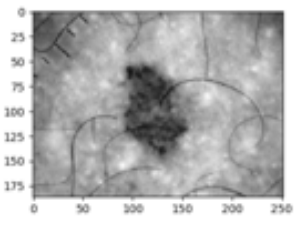

BLUE
Fig. 1. RGB color channels of a skin lesion.

of the segmentation step, deep learning automatically detects the skin lesion and learns a feature representation from a large number of skin images.

In paper [10], an automatic detection of melanoma lesion on skin images has been proposed based on the concept of deep learning. The results shows that deep learning using CNN is able to detect the melanoma lesion efficiently when using CNN with $15 \times 15$ training input size. This neural network architecture can accurately detect melanoma or any kind of skin lesions though.

\section{PROPOSED METHOD}

Generally people tend to see doctors when the melanoma is in its last stage and when it becomes difficult to be treated. People normally consider skin lesion as normal diseases such any environment infection. Thus it becomes a necessity to do self-examination of any suspicious lesion. Nowadays the medical fields depend not only on the doctor diagnosis but also upon computer-aided diagnosis. Digital image processing makes dermatologist and patients life easier. It helps in diagnosing the lesion area without any physical contact with skin, in this section we present our proposed methods that help people and doctors in accurately diagnosing melanoma.

One typical problem in melanoma detection is to separate the skin lesion from the healthy skin. The segmentation step is incredibly sensitive to the output of the preprocessing phase. Thus improving the image analysis and preprocessing by removing the artifacts will certainly improve the segmentation process. Artifacts may occlude some information of the inspected lesion. Artifacts can be hair pixels which should be removed for a better segmentation and lesion analysis. In fact, in the literature many hair removal systems are introduced to deal with that issue.

The preprocessing method for the hair detection proposed in [1] is adopted. A minor changes was introduced. The proposed hair removal system involves several steps which are described below. First, a color enhancement technique based on the blue component of the RGB color channels is selected for the rest of processing which gives better results of both hair removal and segmentation. Fig. 1 shows the differences of gray level values over the 3 RGB color channels. Detecting hair lines from the dermoscopic images is done based on the 2-D derivatives of Gaussian(DOG) of the blue component of the images. The DOG accurately detects lines of the hair in four different directions. Thresholding is then applied to separate the hair 

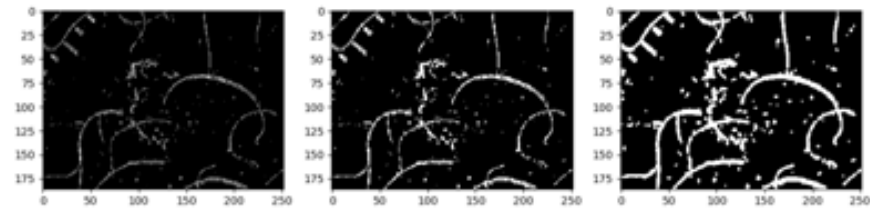

Fig. 2. Hair lines detection using DOG filter.
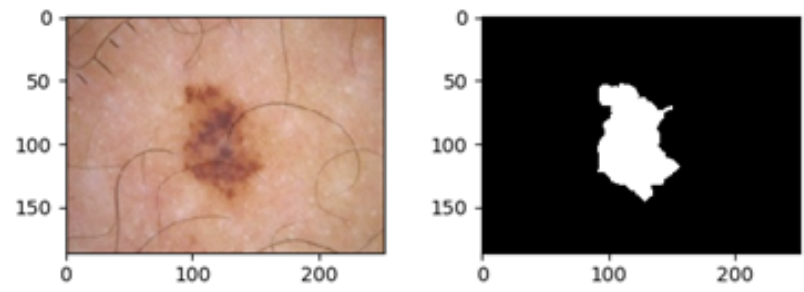

Fig. 3. Skin lesion segmentation using thresholding.

lines from the background. Otsu method is used. Finally, to enhance and refine hair lines, a morphological dilation is introduced(see Fig. 2). The hair mask is filtered one more to remove the unwanted objects by eliminating all the objects that has circular shape and have an area smaller than a threshold. A lesion segmentation process was done to initially recognize the lesion's borders, this step is done by thresholding the images and selecting the region with the largest area(see Fig. 3). This step can approximately give us an information about those pixels belonging to the skin lesion. After that, a pixel classification is done using hierarchical clustering to associate those pixels that does not belong to hair lines neither to the skin lesion. Based on that clustering, a new hair mask is defined and an inpainting process is done. The inpainting algorithm used at that stage is the one proposed by [16] which repairs the hair line pixels of the images based on the neighboring pixels( see Fig. 4). Still, we need to ameliorate these methods by exploring different hair removal methods together. For the lesion segmentation, we used morphological snakes which are faster and numerically more stable than the traditional snakes. There are two Morphological Snakes methods: Morphological Geodesic Active Contours (MorphGAC) and Morphological Active Contours without Edges ( MorphACWE).

MorphACWE [5] works well when the pixel values of the inside and the outside regions of the object to segment have
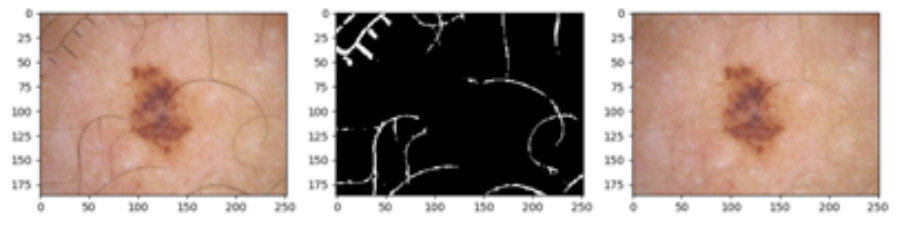

Fig. 4. Final hair mask and image preprocessing result.

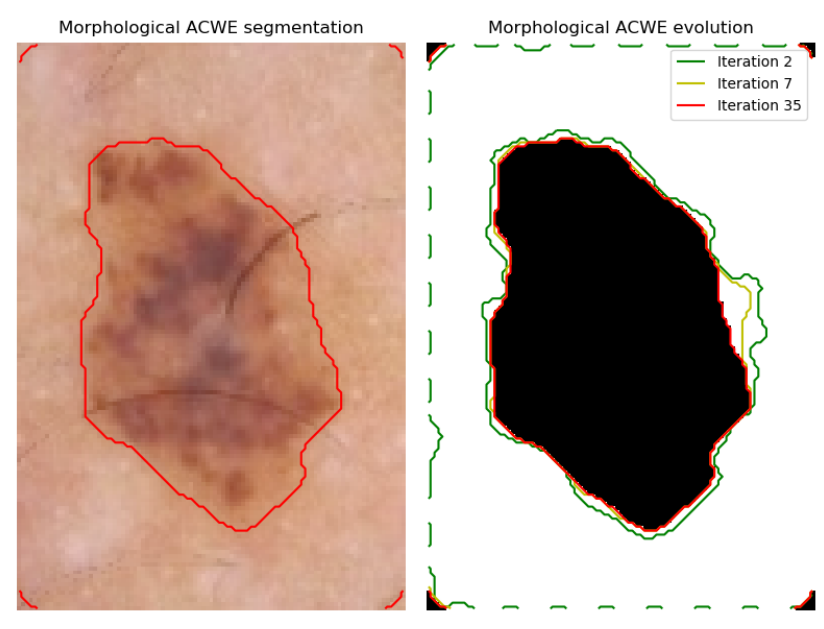

Fig. 5. Skin lesion segmentation using MorphGAC.

different averages. The advantage of MorphACWE is that the contour of the skin lesion is unnecessarily defined( see Fig. 5). However, the search area for the lesion is minimized to make faster and relevant this process. The dimensionality reduction is done based on the result of the initial segmentation which uses thresholding. After extracting the skin lesion, lowlevel features from three visual components: the texture ( SIFT, HOG) descriptors, color (Color Name (CN), colorSIFT) features and other features describing the border of the lesion( convexity, circularity, irregularity index), are extracted and used as descriptors characterizing both benign and malignant lesions. For the training process, an SVM classifier is trained. Also, we used a KNN classifier which find the $\mathrm{K}$ nearest neighbors of an inspected lesion and based on the nature of the majority of neighbors, the lesion is classified whether a malignant or benign.

However, recently many methods using CNNs have been proposed for solving this kind of problem. For that, we tested our chosen dataset using a CNN( Convolutional Neural Network ) architecture. The experiments have been performed using a training input size of 512 lesion images. However, CNNs have to be trained using a huge dataset, This problem has been solved using the data augmentation which is a technique that can be used to artificially expand the size of a training lesion images to efficiently train the model.

The proposed CNN architecture takes as inputs the whole images sized $124 \times 124$ pixels, a preprocessing is needed before training the model. The $\mathrm{CNN}$ architecture is composed of 9 layers( see Fig. 6). Three convolution layers are used( filter size is $3 \times 3$ ) with ReLU activation function, proceeded by three spatial max pooling layers which down-samples the feature maps. The convolution layers aim to learn a feature representation of the input intensity patch, which typically consists of a combination of linear and nonlinear operations, i.e., convolution operation and activation function. The best 


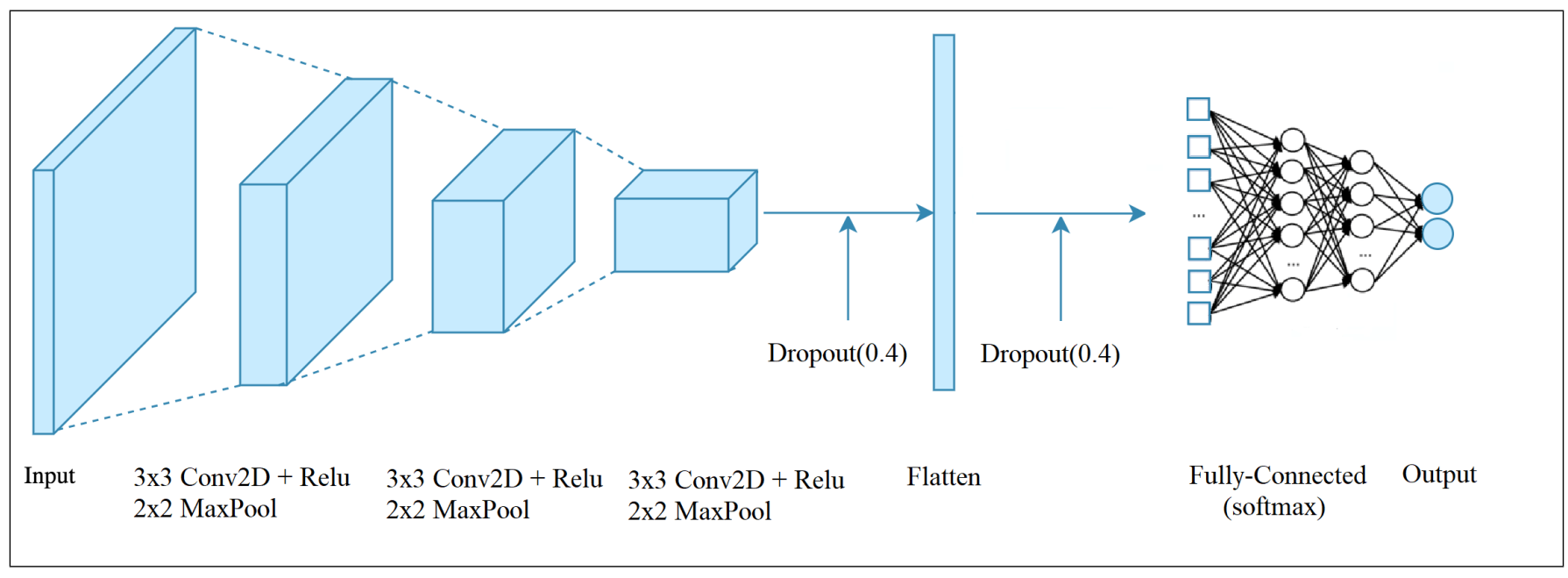

Fig. 6. The $\mathrm{CNN}$ architecture.

solution for reducing overfitting is to obtain more training data which is not practical in medical imaging due to the lack of labeled data. The other solutions include regularization with dropout which is a recently introduced regularization technique. Thus, we introduced to the $\mathrm{CNN}$ model two dropout layers. After extracting the features by the convolution layers and down-sampled by the pooling layers, they are mapped by a fully connected layer with a softmax activation function. The final outputs of the network is the probabilities of a lesion to be either malignant or benign.

\section{EXPERIMENTS AND RESULTS}

The experiments were conducted using a public dataset which is collected from the ISIC(International Skin Imaging Collaboration) archive, it contains more than 23000 images of melanoma. We chose to work with only 640 skin lesion images, containing benign and malignant lesions. They were collected from a dermatoscopy tool. 512 of these images will be used as a training set, the rest as a testing set. Fig. 7 shows some examples of the skin lesion images. The CNN was trained using 640 images of size $124 \times 124$ using the 3 different methods. The CNN was trained for 10 epochs. It is shown that the $\mathrm{CNN}$ has the highest performance over the other two methods, although, classical machine learning and image processing techniques has a few advantages and could also detect melanoma when a $\mathrm{CNN}$ can not. In this matter, a result aggregation of the different method is used in order to ameliorate the performance of the melanoma detection system. Actually, the fusion of multiple model prediction has appeared as an accurate strategy to ameliorate the performance of many pattern recognition systems, rather than relying on one single model prediction. In this paper, we use the majority voting approach which is one of the basic and intuitive approaches. It assigns a sample based on the most frequent class assignment [4].

Table. I summarizes the results of the three proposed method as well as the result of fusing them. Using the KNN classifier,

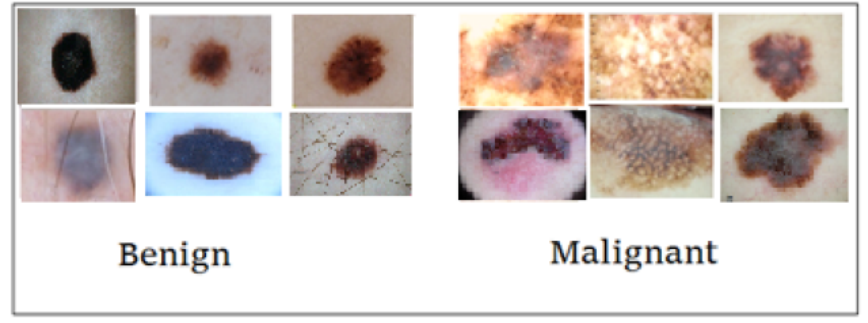

Fig. 7. Image samples of melanoma skin cancer from the ISIC dataset.

TABLE I

THE ACCURACY VALUES OF USING THE DIFFERENT METHODS

\begin{tabular}{|c|c|c|c|c|}
\hline Methods & KNN & SVM & CNN & Majority voting \\
\hline Accuracy & $57.3 \%$ & $71.8 \%$ & $85.5 \%$ & $88.4 \%$ \\
\hline
\end{tabular}

we obtained the lowest accuracy considering the 5 nearest neighbors only. KNN barely can identify malignant skin lesions since it is sensitive to outliers. However, SVM classifier performs better than KNN due to its efficiency and adaptability. Although an SVM classifier achieved a quiet performance, still the CNN is considered as a more powerful and robust tools for identifying melanoma skin cancer. Yet, fusing the decision of all these systems obviously can ameliorate their performance, the idea is to take the decision not based on one single result but on many results.

\section{CONCLUSION AND DISCUSSION}

Melanoma is the deadliest form of skin cancer, can be a non life-threatening cancer if it is diagnosed at an early stage. Thus, it becomes vital to use supportive imaging techniques that have been shown to improve and facilitate the diagnosis process. These techniques are build based on strategies invented by physicians to capture the melanoma at an early stage. We Have introduced a hybrid method for melanoma skin cancer detection that can be used to examine any suspicious lesion 
in this paper. Our proposed system rely on combining the prediction of three different methods using majority voting. Most of the systems rely only on using two rules defined by physicians: the $\mathrm{ABCD}$ and the Blue-Black rule, which are shown to have some limitations and proved ineffective in some cases. Another concept was proposed which is the ugly duckling. The idea is to observe not only the morphology of the lesion in question, but also to compare it to that of surrounding lesions, looking for an outlier in the background of similar-appearing moles. This clue was clinically proved to be a good criteria to detect melanoma, but it has not been explored in the automatic melanoma detection systems yet. Thus, an idea of finding the weird skin lesion among a set of lesions could be explored in a further study. Those who work on the field of medical imaging has been suffered from a very serious issue which is the lack of enough labeled data to train their systems. Thus, the use of semi-supervised learning could be used to cover this issue.

\section{REFERENCES}

[1] Qaisar Abbas, M Emre Celebi, and Irene Fondón García. Hair removal methods: a comparative study for dermoscopy images. Biomedical Signal Processing and Control, 6(4):395-404, 2011.

[2] Giuseppe Argenziano, Caterina Longo, A Cameron, S Cavicchini, J-Y Gourhant, A Lallas, I McColl, C Rosendahl, L Thomas, D TiodorovicZivkovic, et al. Blue-black rule: a simple dermoscopic clue to recognize pigmented nodular melanoma. British Journal of Dermatology, 165(6):1251-1255, 2011

[3] Samy Bakheet. An svm framework for malignant melanoma detection based on optimized hog features. Computation, 5(1):4, 2017.

[4] D Ballabio, R Todeschini, and V Consonni. Recent advances in highlevel fusion methods to classify multiple analytical chemical data. In Data Handling in Science and Technology, volume 31, pages 129-155. Elsevier, 2019.

[5] Tony F Chan and Luminita A Vese. Active contours without edges IEEE Transactions on image processing, 10(2):266-277, 2001.

[6] Fekrache Dalila, Ameur Zohra, Kasmi Reda, and Cherifi Hocine. Segmentation and classification of melanoma and benign skin lesions. Optik, 140:749-761, 2017.

[7] Florentia Dimitriou, Regina Krattinger, Egle Ramelyte, Marjam J Barysch, Sara Micaletto, Reinhard Dummer, and Simone M Goldinger. The world of melanoma: epidemiologic, genetic, and anatomic differences of melanoma across the globe. Current oncology reports, 20(11):87, 2018.

[8] Diwakar Gautam and Mushtaq Ahmed. Melanoma detection and classification using svm based decision support system. In 2015 Annual IEEE India Conference (INDICON), pages 1-6. IEEE, 2015.

[9] Tsong-Long Hwang, Woan-Ruoh Lee, Shu-Chiou Hua, and Jia-You Fang. Cisplatin encapsulated in phosphatidylethanolamine liposomes enhances the in vitro cytotoxicity and in vivo intratumor drug accumulation against melanomas. Journal of dermatological science, 46(1):1120, 2007.

[10] Ashwin R Jadhav, Arun G Ghontale, and Vimal K Shrivastava. Segmentation and border detection of melanoma lesions using convolutional neural network and svm. In Computational Intelligence: Theories, Applications and Future Directions-Volume I, pages 97-108. Springer, 2019.

[11] Uzma Jamil, Asma Sajid, Majid Hussain, Omer Aldabbas, Afshan Alam, and M Umair Shafiq. Melanoma segmentation using bio-medical image analysis for smarter mobile healthcare. Journal of Ambient Intelligence and Humanized Computing, 10(10):4099-4120, 2019.

[12] JC Kavitha, A Suruliandi, D Nagarajan, and T Nadu. Melanoma detection in dermoscopic images using global and local feature extraction. International Journal of Multimedia and Ubiquitous Engineering, 12(5):19-28, 2017.
[13] Zaher Khazaei, F Ghorat, AM Jarrahi, HA Adineh, M Sohrabivafa and E Goodarzi. Global incidence and mortality of skin cancer by histological subtype and its relationship with the human development index (hdi); an ecology study in 2018. World Cancer Research Journal, 6:13, 2019.

[14] Ilias Maglogiannis and Charalampos N Doukas. Overview of advanced computer vision systems for skin lesions characterization. IEEE transactions on information technology in biomedicine, 13(5):721-733, 2009.

[15] Dirk Schadendorf, Alexander CJ van Akkooi, Carola Berking, Klaus G Griewank, Ralf Gutzmer, Axel Hauschild, Andreas Stang, Alexander Roesch, and Selma Ugurel. Melanoma. The Lancet, 392(10151):971984, 2018.

[16] Alexandru Telea. An image inpainting technique based on the fast marching method. Journal of graphics tools, 9(1):23-34, 2004.

[17] Jufeng Yang, Xiaoxiao Sun, Jie Liang, and Paul L Rosin. Clinical skin lesion diagnosis using representations inspired by dermatologist criteria. In Proceedings of the IEEE Conference on Computer Vision and Pattern Recognition, pages 1258-1266, 2018.

[18] Chanki Yu, Sejung Yang, Wonoh Kim, Jinwoong Jung, Kee-Yang Chung, Sang Wook Lee, and Byungho Oh. Acral melanoma detection using a convolutional neural network for dermoscopy images. PloS one, 13(3), 2018 